\title{
Lithium-Ionen-Batterien - Kreislaufwirtschaftliche Herausforderungen am Ende des Lebenszyklus und im Recycling
}

\author{
Thomas Nigl'1, Bettina Rutrecht' ${ }^{1}$, Michael Altendorfer ${ }^{1}$, Silvia Scherhaufer ${ }^{2}$, Ina Meyer ${ }^{3}$, Mark Sommer ${ }^{3}$ und \\ Peter Beigl ${ }^{2}$
}

\begin{abstract}
${ }^{1}$ Lehrstuhl für Abfallverwertungstechnik und Abfallwirtschaft, Montanuniversität Leoben, Leoben, Österreich ${ }^{2}$ Institut für Abfallwirtschaft, Universität für Bodenkultur Wien, Wien, Österreich ${ }^{3}$ Österreichisches Institut für Wirtschaftsforschung, Wien, Österreich
\end{abstract}

Eingegangen 18. Januar 2021; angenommen 26. Januar 2021; online publiziert 19. Februar 2021

\begin{abstract}
Zusammenfassung: Mit anhaltender Verkehrswende nimmt die Bedeutung von Lithium-Ionen-Batterien (LIB) in Zukunft stark zu. Im Sinne der Kreislaufwirtschaft sind einerseits die optimale und nachhaltige Nutzung vorhandener Ressourcen, andererseits das Schließen von Material-Kreisläufen durch geeignete und effiziente Recyclingverfahren unabdingbar. Der Artikel behandelt die aktuellen Problemfelder und Herausforderungen entlang der Wertschöpfungskette von LIB am Ende ihres Lebenszyklus. Dabei werden insbesondere Aspekte der Vorbehandlung und des Recyclings beleuchtet.
\end{abstract}

Schlüsselwörter: Second Life, Lebenszyklus, Vorbehandlung

Lithium-Ion Batteries-Circular Economy-related Challenges at the End-of-Life and in Recycling

Abstract: The ongoing shift to low carbon mobility schemes will significantly increase the deployment of lithium-ion batteries (LIB) in the future. In terms of the circular economy, the optimal and sustainable use of existing resources, on the one hand, and the closing of material cycles through suitable and efficient recycling processes, on the other hand, are inevitable. The article deals with the current problem areas and challenges along the value chain of LIBs at the end of their life cycle. In particular, it deals with aspects of pre-treatment and recycling.

\section{Keywords: Second Life, Life cycle, Pre-treatment}

\section{T. Nigl ( $\triangle)$}

Lehrstuhl für Abfallverwertungstechnik und Abfallwirtschaft,

Montanuniversität Leoben,

Franz-Josef-Straße 18,

8700 Leoben, Österreich

thomas.nigl@unileoben.ac.at

\section{Einleitung}

Lithium-lonen-Batterien (LIB) haben in den letzten Jahren eine zentrale Rolle in der Verkehrswende und in der Reduktion von Treibhausgasemissionen im Verkehrsbereich eingenommen. Dazu kommen die immer stärkere Verwendung von LIB im Bereich von stationären Energiespeichern (u.a. als Puffersysteme in Kombination mit erneuerbaren Energieträgern) und ein ungebrochener Trend zur weiteren Marktdurchdringung im Elektronikbereich.

Diese Entwicklung ist begleitet von einem steigenden Ressourcenbedarf in der Produktion und von technischen Herausforderungen im End-of-Life (EoL). Aus dem starken Wachstum und der zeitlichen Verzögerung durch die Nutzungsphase ergibt sich eine hohe Bedarfslücke, die durch Sekundärressourcen nicht zu decken ist.

Im Hinblick auf endliche Ressourcen und eine funktionierende Kreislaufwirtschaft ist jedoch eine möglichst effektive und effiziente Nutzung von Sekundärressourcen erstrebenswert. Folgende Maßnahmen sind dazu geeignet, dieses Ziel zu erreichen:

1. Lange Nutzungsphasen durch langlebige Produkte

2. Verlängerung der Nutzungsphase durch Reparatur

3. Wiederverwendung im Sinne von Second Use

4. Optimierte Recyclingverfahren bzw. eine hohe Recyclingquote

Aktuelle Zahlen aus Österreich und Deutschland zeigen enorme Steigerungen im Anteil von vollelektrischen und hybriden Antriebssystemen [1, 2]. Im Vorreiterland Norwegen haben KFZ mit vollelektrischem Antrieb 2020 bei den Neuzulassungen einen Anteil von 54,3\% erreicht, dazu kommen weitere $20,4 \%$ mit Plug-in-Hybrid-Antrieb [3].

Je nach zugrundeliegendem Rechenmodell kann in Zukunft mit sehr unterschiedlichen Zuwachsraten gerechnet 


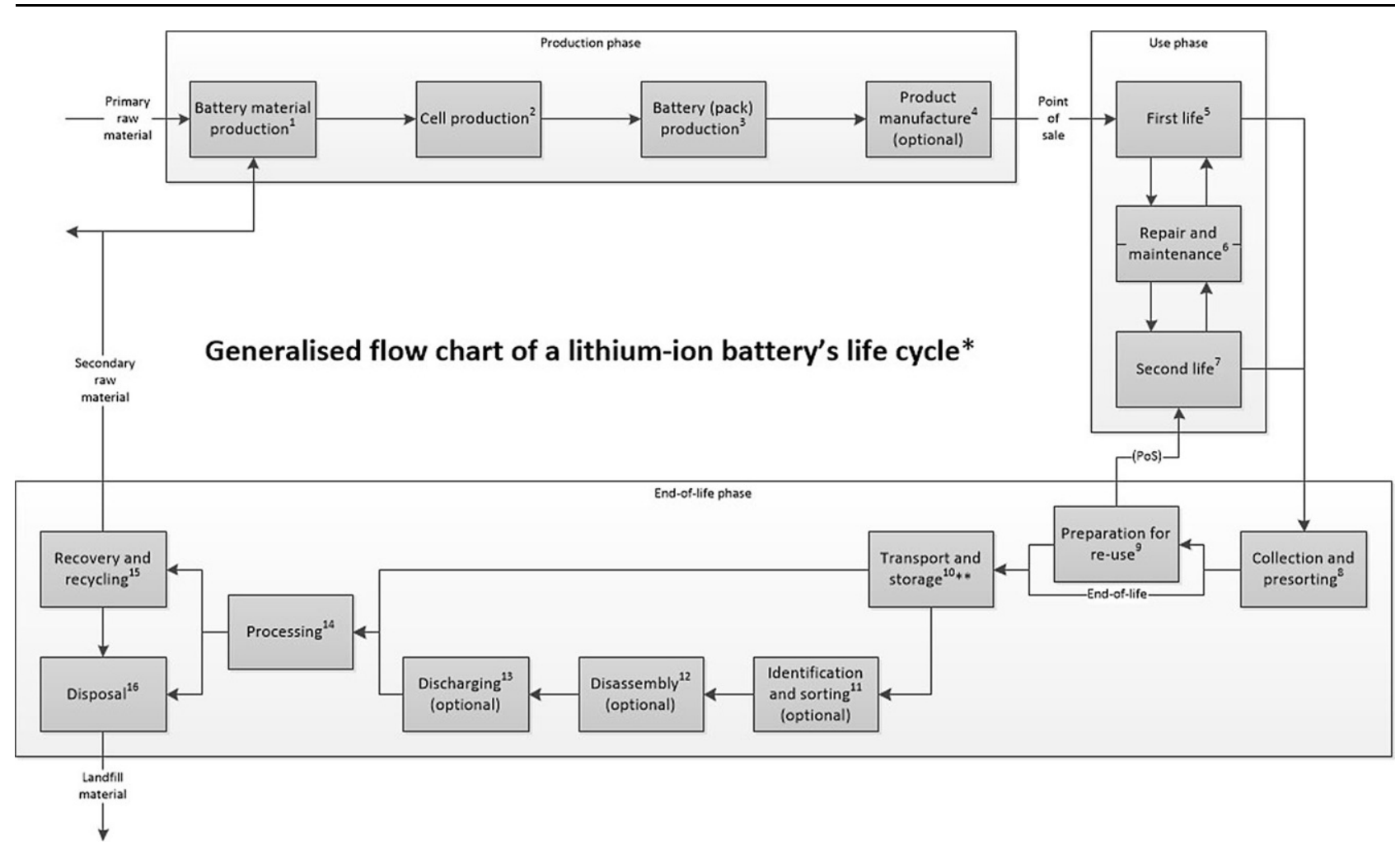

Abb. 1: Schematische Darstellung der Wertschöpfungskette für Lithium-lonen-Batterien

werden [4, 5]. Im Gegensatz zu den beträchtlichen Zuwächsen im Elektromobilitätssektor erreichen aktuell nur bescheidene Mengen an Elektrofahrzeugen das Ende ihres Lebenszyklus. Die aktuelle Zusammensetzung der ausgedienten LIB ist daher sehr stark von mobilen Elektro- und Elektronikgeräten und E-Bikes geprägt [4].

In Abb. 1 ist eine schematische Wertschöpfungskette von LIB im Sinne der Kreislaufwirtschaft mit den einzelnen Lebenszyklusphasen Produktions-, Nutzungs- und End-ofLife-Phase dargestellt.

\section{Kreislaufwirtschaftliche Heraus- forderungen}

Im Folgenden werden beispielhaft die aktuellen Hemmnisse und Herausforderungen, denen sich die Sekundärrohstoffindustrie im Bereich Batterierecycling zurzeit stellt, diskutiert. Die Auflistung bezieht sich vor allem auf den Bereich der aus Sicht der Abfallwirtschaft im EoL angekommenen Batterien und deren Recycling und schließt explizit ökosoziale Themen aus der Nutzungsphase (z. B. Reparaturdienstleistungen, Sharing) sowie die Schnittstelle Sammlung und Sammelsysteme aus.

\subsection{Sicherheit}

LIB stellen aufgrund ihrer hohen Energiedichte in allen Bereichen sicherheitstechnische Herausforderungen dar, welche sich je nach Lebenszyklusphase sehr stark unterschei- den können. Bei den LIB im EoL stehen Gefahren durch mechanische oder thermische Beschädigungen im Vordergrund [6].

Um die Sicherheit von LIB im Transport zu gewährleisten, gab es in den letzten Jahren regelmäßige Anpassungen und Verschärfungen von Vorschriften im ADR, dem internationalen Abkommen zum Transport von gefährlichen Abfällen auf der Straße. Spezifische Verpackungsvorschriften sollen ein thermisches Durchgehen und Ansteckung weiterer Batterien verhindern.

\subsection{Beurteilung bzw. Bewertung von LIB im End- of-Life}

Die umfangreiche mediale Berichterstattung zu einzelnen spektakulären Unfällen mit Elektrofahrzeugen hat zu einer stärkeren öffentlichen Wahrnehmung der Gefahren geführt. Doch nicht nur im Falle eines Unfalls ist die Bewertung des Zustands der Batterien aus sicherheitstechnischer Sicht von Belang, sondern auch wenn eine Nachnutzung im Sinne eines Second Life (bspw. als stationärer Batteriespeicher) vorgesehen ist.

An Best-Practice-Lösungen wird gerade branchenweit gearbeitet, bisher sind keine Standards und Kriterien für weitere Verwendung vorhanden. 


\subsection{Second Life}

Die Umsetzung zahlreicher Pilotprojekte unterschiedlicher Größenordnungen demonstriert einerseits die Machbarkeit von stationären Batteriespeichern aus Second-Life-Batterien, andererseits das große Potenzial an Anwendungsbereichen $[7,8]$. Inwiefern ein Hochskalieren bzw. großtechnische Anlagen auch ohne Förderungen umgesetzt werden können, ist noch nicht beantwortet. Herausforderungen sind einerseits die logistische Beschaffung und andererseits die aufwändige Prüfung geeigneter und miteinander kompatibler EoL-Batterien. Datenschutzrechtliche Probleme in Bezug auf Nutzungsdaten vom ersten Leben (z. B. im Elektroauto) zur Beurteilung der Batterielebensdauer im zweiten Leben sowie rechtliche Rahmenbedingungen, wie der Haftungsübergang vom Erstproduzenten zu SecondLife-Verkäufern, sind ebenso noch zu klären.

\subsection{Vorbehandlung}

\subsubsection{Erkennung und Sortierung}

Die (automatische oder manuelle) Erkennung und Sortierung von LIB in die einzelnen Subtypen ist problematisch bzw. zeitaufwändig, da die Batterien in der Regel unzureichend gekennzeichnet sind. Zwar gibt es seit 2019 einen Standard [9] zur einheitlichen Kennzeichnung, dieser unterscheidet aber nur grundlegende elektrochemische Systeme (z. B. Bleisäure-, Nickelmetallhydrid-, Lithium-lonen-Batterien). Doch unter Lithium-Ionen-Batterien sind nochmals eine Vielzahl elektrochemischer Systeme zusammengefasst (z. B. Lithium-Eisenphosphat, Lithium-Cobaltoxid, LithiumNickel-Mangan-Cobaltoxid), die sich anhand der verwendeten Aktivmaterialien - feinpulvrige Lithiummetalloxide maßgeblich voneinander unterscheiden. Das Aktivmaterial macht bis zu $60 \mathrm{Gew}$.-\% der Zelle aus [10] und eine getrennte Behandlung verschiedener LIB-Typen ist daher essentiell für das Recycling und die Erfüllung der vorgeschriebenen Recyclingquote.

\subsubsection{Demontage}

Durch Demontage können einfach erreichbare, wertvolle und gut recycelbare Bauteile vor dem tatsächlichen Recyclingschritt entfernt werden. Aus Gehäusedeckel, Kabelstrang, Kühlsystem und elektronischen Bauteilen werden so einerseits wieder Wertstoffe wie Stahl, Kupfer, ausgewählte Kunststoffe und Edelmetalle zurückgewonnen und andererseits verringert sich der zu behandelnde Volumenstrom. Das spart Energie und Investitionskosten.

Als Herausforderungen im Bereich der Demontage sind der hohe Personal- und Zeitaufwand [11, 12], die notwendige Fachausbildung für Arbeiten im Hochvoltbereich, die mangelnde Normbauweise und die damit zusammenhängenden hohen Investitionskosten für Automatisierungstechnik [13] zu nennen.

\subsubsection{Entladung}

Ein niedriger Ladestand der Batterie ist bei der weiteren mechanischen Aufbereitung von großen Energiespeichersystemen von sicherheitstechnischem Vorteil $[14,15]$ und wird bei fast allen Recyclingverfahren angewandt, da sonst die in den Zellen vorhandene Energie einen Mehraufwand hinsichtlich Kühlung bedeutet [16]. Ausnahmen davon sind Verfahren mit Kryotechnologie, Inertgasspülung und die direkte pyrometallurgische Aufarbeitung auf Zell- bzw. Modulebene im Schachtofen (z. B. Fa. Umicore).

Als Stand der Technik gilt die Entladung über einen Ohm'schen Widerstand und wird bei Entsorgungsfachbetrieben vor allem bei Energiespeichern aus Elektrofahrzeugen durchgeführt. Die Energie wird dabei entweder in Form von Wärme abgeführt oder gespeichert.

Für die Entladung bestehen aus ähnlichen Gründen die gleichen Nachteile wie für die Demontage: Mangelnde Automatisierbarkeit, die Artenvielfalt von Batteriemodulen und die notwendige Fachausbildung des Personals treiben die Kosten des Recyclings in die Höhe. Eine wissenschaftliche Aussage über eine durchschnittlich zurückgewinnbare Energie gibt es noch nicht. Dennoch kann davon ausgegangen werden, dass sich in Summe eine potenzielle Kosteneinsparung durch kluge Rückeinspeisung zu Hochlastzeiten beziehungsweise durch Skalierung der Batteriemenge ergibt.

\subsection{Recyclingverfahren}

Im Zuge der aktuell stattfindenden Überarbeitung der Batterierichtlinie der EU ist davon auszugehen, dass die Recyclingquote für LIB erhöht wird. Im vorliegenden RichtlinienEntwurf wird vorgeschlagen, 2025 bzw. 2030 die aktuell für LIB bei $50 \%$ liegende Recyclingquote auf 65 bzw. $70 \%$ anzuheben und um weitere Zielvorgaben auf elementarer Ebene zu ergänzen (90 bzw. $95 \%$ für $\mathrm{Co}, \mathrm{Ni}, \mathrm{Cu}$ und 35 bzw. $70 \%$ für Li) [17]. Damit erhöhen sich auch die Anforderungen an bestehende und zukünftige Recyclingverfahren und der Druck, endlich den Brückenschlag zu einem geschlossenen Kreislaufsystem zu schaffen, indem aus Batterien wieder Batterien werden.

LIB weisen dabei typische Merkmale von Future waste auf [18]: (1) Sie bieten wissenschaftliche oder technische Vorteile, (2) sind aus technischer Sicht komplex und (3) gehen erst seit kürzerer Zeit in den anthropogenen Bestand ein.

Letzteres führt in der Regel dazu, dass Recyclingpfade für den entsprechenden Future waste noch nicht vollständig etabliert bzw. ausgereift sind. Obwohl es in den letzten Jahren eine Fülle an Forschungs- und Entwicklungsprojekten zu EoL-LIB gab, befinden sich viele Anlagen erst im Versuchs- bzw. Pilotmaßstab oder gerade in industrieller Umsetzung.

In Summe bestehen sie alle aus einer Kombination von verschiedenen Verfahrensschritten mit unterschiedlich starker Ausprägung und Gewichtung. Dazu gehören: 
- die thermische Konditionierung,

- die mechanische Aufbereitung,

- die pyrometallurgische Aufarbeitung,

- die hydrometallurgische Aufarbeitung,

- das direkte Recycling.

Jeder Weg bringt seine eigenen Vorteile, verknüpft mit individuellen Herausforderungen, denen sich die Betreiber stellen müssen.

\subsubsection{Thermische Konditionierung}

Der Pyrolyseprozess findet bei etwa $500^{\circ} \mathrm{C}$ statt. Durch die thermische Belastung schmilzt der Separator ab und es kommt zu einem Kurzschluss, welcher weitere Zersetzungsreaktionen mit sich bringt - ein sogenannter Thermal Runaway [19]. Dadurch kommt es zu einer schlagartigen Zunahme des Abgas-Volumenstroms, welcher jedoch konstant sein sollte [20]. Zudem entstehen fluorhaltiges Benzol und Ester-Elektrolyt, welche durch Kondensation gesammelt werden [21]. Steigen die Temperaturen über $660^{\circ} \mathrm{C}$, so oxidiert ein Großteil des Aluminiums, was die Ausbeute im Recyclingprozess reduziert [19].

\subsubsection{Mechanische Aufbereitung}

Für die mechanische Aufbereitung müssen die Batterien tiefenentladen werden. Bei der Zerkleinerung wird der Elektrolyt freigesetzt. Dieser zeigt eine hohe Reaktivität mit Sauerstoff und beinhaltet unter anderem Fluor und andere giftige Stoffe [22]. Der Prozess muss unter inerter Atmosphäre $\left(z \mathrm{~B} . \mathrm{CO}_{2}\right)$ oder weit unter Raumtemperatur stattfinden, da es sonst zu Explosionen oder Brand kommen kann [23]. Zudem sollte der Zerkleinerungsapparat korrosionsbeständig sein, da das Leitsalz im Elektrolyt zu Fluss- oder Phosphorsäure reagiert. Eine weitere Herausforderung stellt die Trennung der Beschichtung von den Ableiterfolien dar [20].

\subsubsection{Pyrometallurgische Aufbereitung}

Im pyrometallurgischen Prozess erfolgt eine Rückgewinnung von nur wenigen wertvollen Metallen (Nickel, Kobalt und Kupfer). Als Produkt entsteht eine Legierung, die in weiterer Folge meist hydrometallurgisch aufbereitet wird [24]. Lithium, Mangan und Aluminium verbleiben in der Schlacke, deren Rückgewinnung kostenintensiv und mit aufwendigen Prozessen verbunden ist [19, 25, 26]. Die Anode, der Elektrolyt und die Kunststoffe dienen als Brennstoff [24]. Zusätzlich benötigt die pyrometallurgische Aufbereitung viel Energie und erzeugt umweltschädliche Abgase, die einer Behandlung bedürfen [26].

\subsubsection{Hydrometallurgische Aufbereitung}

Beim hydrometallurgischen Prozess erfolgt die Metallrückgewinnung mittels wässriger Chemie. In diesem Verfahren werden Säuren oder Basen zur Auslaugung verwendet, danach erfolgt die Aufkonzentrierung und Reinigung [24]

Dazu sind eine Vorbehandlung der eingebrachten Batterien und ein stabiler Input notwendig. Zudem kann die Auf- trennung in die einzelnen Elemente aufwendig sein. Nachteilig sind auch die lange Verweildauer in der Prozesskette und die kostspielige Abwasseraufbereitung [24, 26].

\subsubsection{Direktes Recycling}

Diese Methode befindet sich noch im Labor-bzw. Pilotmaßstab [24]. Die Trennung der einzelnen Batteriebestandteile erfolgt meist mit mechanischen Prozessen oder einer moderaten thermischen Verarbeitung [24, 27, 28], wobei sich hier ebenso die dazu erwähnten Herausforderungen zeigen.

Zudem bedarf es einer extrem genauen Sortierung nach der Kathodenchemie, was den Prozess unflexibel und sehr empfindlich auf Veränderungen im Inputstrom macht. Auch der Lithiumverslust kann nicht allgemein für alle Batterien gleichermaßen angenommen werden. Daher ist es schwierig, rezykliertes Material mit exzellenter elektrochemischer Performance zu produzieren, die der Batterieerzeugung genügen $[24,28]$.

\subsubsection{Weitere Herausforderungen}

LFP-Batterien stellen nahezu alle existierenden Recyclingverfahren vor große Herausforderungen, da die enthaltenen Phosphatverbindungen den Prozessablauf stören. Eigene Recyclinglösungen für LFP-Batterien existieren noch nicht bzw. sind gerade Gegenstand der Forschung [29].

Ähnliches gilt für die Rückgewinnung von Lithium. Das sehr unedle Metall geht in pyrometallurgischen Prozessen in die Schlacke und kann daraus nur schwer rückgewonnen werden. Hydrometallurgische Prozesse sind aktuell Gegenstand der Forschung [30].

\section{Fazit}

Die Kreislaufwirtschaft ist gesellschaftspolitischer Konsens und die logische Antwort auf das Bedürfnis für einen nachhaltigen Umgang mit endlichen Ressourcen. Um sie für Lithium-lonen-Batterien zu erreichen, besteht noch einiger Handlungs- und Entwicklungsbedarf.

Eine stärkere europaweite Vernetzung und Zusammenarbeit von Stakeholdern, entlang der Wertschöpfungskette und über nationale Landesgrenzen hinaus, ist aus Sicht kritischer Rohstoffe und geopolitischer Abhängigkeiten notwendig. Die neue Batterierichtlinie definiert dabei den wirtschaftspolitischen Rahmen für zukünftige Investitionen in LIB-Recycling.

Ein Second Life von LIB, beispielsweise als stationärer Energiespeicher, ist technisch möglich und kann die Nutzungsdauer von Batterien verlängern. Dies spart einerseits Ressourcen in der Produktion sowie Energie in der Verarbeitung von Batterien und kann andererseits ein technisch fortgeschrittenes Recycling zu einem späteren Zeitpunkt ermöglichen

Eine wissenschaftliche Beurteilung der Rentabilität und der ökologischen sowie ökonomischen Performance von Vorbehandlung- und Recyclingverfahren ist essentieller Begleiter zukünftiger Entwicklungen, um einerseits zu erwar- 
tende Prozessoptimierungen vergleichbar zu machen und andererseits das Hochskalieren von Recyclingkapazitäten im kommenden Jahrzehnt zu monitoren.

Förderung. Das Projekt, Developing the Lithium-Ion Battery Value Chain fo Recycling in Austria' wird vom Klima- und Energiefonds (Förderaktion ,Zero Emission Mobility') gefördert.

Funding. Open access funding provided by Montanuniversität Leoben.

Open Access Dieser Artikel wird unter der Creative Commons Namensnennung 4.0 International Lizenz veröffentlicht, welche die Nutzung, Vervielfältigung, Bearbeitung, Verbreitung und Wiedergabe in jeglichem Medium und Format erlaubt, sofern Sie den/die ursprünglichen $\mathrm{Au}$ tor(en) und die Quelle ordnungsgemäß nennen, einen Link zur Creative Commons Lizenz beifügen und angeben, ob Änderungen vorgenommen wurden.

Die in diesem Artikel enthaltenen Bilder und sonstiges Drittmaterial unterliegen ebenfalls der genannten Creative Commons Lizenz, sofern sich aus der Abbildungslegende nichts anderes ergibt. Sofern das betreffende Material nicht unter der genannten Creative Commons Lizenz steht und die betreffende Handlung nicht nach gesetzlichen Vorschriften erlaubt ist, ist für die oben aufgeführten Weiterverwendungen des Materials die Einwilligung des jeweiligen Rechteinhabers einzuholen.

Weitere Details zur Lizenz entnehmen Sie bitte der Lizenzinformation auf http://creativecommons.org/licenses/by/4.0/deed.de.

\section{Literatur}

1. BEO: E-Autos in Österreich. 2021. URL: https://www.beoe.at/statistik/ (12.01.2020)

2. Statista: Anzahl der Neuzulassungen von Elektroautos in Deutschland von 2003 bis 2020. 2021. URL: https://de.statista.com/statistik/ daten/studie/244000/umfrage/neuzulassungen-von-elektroautosin-deutschland/ (12.01.2020).

3. The Guardian: Electric cars rise to record $54 \%$ market share in Norway, 2021, https://www.theguardian.com/environment/2021/jan/05/ electric-cars-record-market-share-norway (12.01.2020).

4. Beigl, P.; Scherhaufer, S.; Nigl, T.; Meyer, I.; Altendorfer, M.; Part, F.; Sommer, M.; Salhofer, S.; Pomberger, R.: Entwicklung einer vollständigen Wertschöpfungskette für Lithium-lonen Batterierecycling in Österreich. Recy \& DepoTech 2020, 18.-20. November 2020, Montanuniversität Leoben, 2020

5. Meyer, I.; Sommer, M.; Kratena, K.: How to reach Paris: a compre hensive long-term energy-economy scenario for Austria, In Zachariadis, T.; Milne, J. E.; Andersen, M. S.; Ashiabor, H. (Eds.): Economic Instruments for a Low-carbon Future Critical Issues in Environmental Taxation, Cheltenham, UK: Edward Elgar Publishing, 2020, pp 127-140

6. Nigl, T.; Baldauf, M.; Hohenberger, M.; Pomberger, R.: Lithium-lon Batteries as Ignition Sources in Waste Treatment Processes-A Semi-Quantitative Risk Analysis and Assessment of Battery-Caused Waste Fires, Processes, 9 (2021), no 49, https://doi.org/10.3390/ pr9010049

7. Stein, V.; Arnberger, A.; Nickl, A.; Ungerböck, R.; Werinos, M.: Batterien aus der E-Mobilität in Second Life Anwendungen, Recy \& DepoTech 2020, 18.-20. November 2020, Montanuniversität Leoben, 2020

8. Renault: Renault Eways: The Group presents two major new energy storage projects. Press Release from 21 Oct 2020, Groupe Renault Press Office, 2020. URL: https://en.media.groupe.renault.com/ news/renault-eways-the-group-presents-two-major-new-energystorage-projects-6714-989c5.html (12.01.2021).

9. IEC 62902:2019-02: Secondary cells and batteries-Marking symbols for identification of their chemistry

10. Arnberger, A.; Coskun, E.; Rutrecht, B.: Recycling von Lithium-lonen-Batterien. In Thiel, S.; Thomé-Kozmiensky, E.; Goldmann, D. (Hrgb.): Recycling und Rohstoffe, Nietwerder: TKh, 2018
11. Alfaro-Algaba, M.; Ramirez, F. J.: Techno-economic and environmental disassembly planning of lithium-ion electric vehicle battery packs for remanufacturing, Resources, Conservation and Recycling, 154 (2020), 104461, https://doi.org/10.1016/j.resconrec.2019.104461

12. Rallo, H.; Benveniste, G.; Gestoso, I.; Amante, B.: Economic analysis of the disassembling activities to the reuse of electric vehicles $\mathrm{Li}$ ion batteries, Resources, Conservation and Recycling, 159 (2020), 104785. https://doi.org/10.1016/j.resconrec.2020.104785

13. Lyon, T., Mütze, T.; Peuker U.A.: Mechanical processing of lithiumion-batteries in dependence to the dismantling level, Recy \& DepoTech 2020, 18.-20. November 2020, Montanuniversität Leoben. In Pomberger Roland; Adam Josef; Aldrian Alexia; et al (Hrgb.): Konferenzband zur 15. Recy DepoTech-Konferenz, 2020

14. Golubkov, A. W.; Scheikl, S.; Planteu, R.; Voitic, G.; Wiltsche, H.; Stangl, C., Fauler, G.; Thaler, A.; Hacker, V.: Thermal runaway of commercial 18650 Li-ion batteries with LFP and NCA cathodes-impact of state of charge and overcharge, RSC Adv., 5 (2015), no 70, 57171-57186, https://doi.org/10.1039/C5RA05897J

15. Lee, C.; Said, A.O.; Stoliarov, S. I.: Impact of State of Charge and Cell Arrangement on Thermal Runaway Propagation in Lithium lon Battery Cell Arrays. Transportation Research Record, 2673 (2019), no 8, pp 408-417, https://doi.org/10.1177/0361198119845654

16. Harper, G.; Sommerville, R.; Kendrick, E.; Driscoll, L.; Slater, P.; Stolkin, R.; Walton, A.; Christensen, P.; Heidrich, O.; Lambert, S.; Abbott, A.; Ryder, K.; Gaines, L.; Anderson, P.: Recycling lithium-ion batteries from electric vehicles. Nature, 575 (2019), no 7781, pp 75-86; https://doi.org/10.1038/s41586-019-1682-5

17. Europäische Kommission: Proposal for a Regulation of the European Parliament and of the Council concerning batteries and waste batteries, repealing Directive 2006/66/EC and amending Regulation (EU) No 2019/1020. URL: https://ec.europa.eu/environment/waste/ batteries/, (12.01.2020).

18. Pomberger, R.; Ragossnig, A.: Future Waste-Waste Future, Waste Management and Research, 32 (2014), S 89-90

19. Brückner, L.; Frank, J.; Elwert, T.: Industrial Recycling of Lithium-lon Batteries-A Critical Review of Metallurgical Process Routes, Metals, 10 (2020), no 8, p 1107, https://doi.org/10.3390/met10081107

20. Arnberger, A.: Entwicklung eines ganzheitlichen Recyclingkonzeptes für Traktionsbatterien basierend auf Lithium-Ionen-Batterien, Dissertation, Lehrstuhl für Abfallverwertungstechnik und Abfallwirtschaft, Leoben: Montanuniversität, 2016

21. Zhang, G.; He, Y.; Feng, Y.; Wang, H.; Zhang, T.; Xie, W.; Zhu, X.: Enhancement in liberation of electrode materials derived from spent lithium-ion battery by pyrolysis. Journal of Cleaner Production, 199 (2018), pp 62-68, https://doi.org/10.1016/j.jclepro.2018.07.143

22. Pistoia, G.; Liaw, B.: Behaviour of Lithium-Ion Batteries in Electric Vehicles: Battery Health, Performance, Safety and Cost, Springer, 2018, https://doi.org/10.1007/978-3-319-69950-9

23. Vandepaera, L.; Cloutier, J.; Amor, B.: Environmental impacts of Lithium Metal Polymer and Lithium-ion stationary batteries. Renewable and Sustainable Energy Reviews, 78 (2017), pp 46-60, https:// doi.org/10.1016/j.rser.2017.04.057

24. Chen, M.; Ma, X.; Chen, B.; Arsenault, R.; Karlson, P.; Simon, N. Wang, Y.: Recycling End-of-Life Electric Vehicle Lithium-Ion Batteries, Joule, 3 (2019), no 11, pp 2622-2646, https://doi.org/10.1016/j. joule.2019.09.014

25. Hoyer, C.: Strategische Planung des Recyclings von Lithium-lonenBatterien aus Elektrofahrzeugen in Deutschland, Dissertation Technische Universität Braunschweig, Springer, 2015, https://doi.org/10. 1007/978-3-658-10274-6

26. Zheng, X.; Zhu, Z.; Lin, X.; Zhang, Y.; He, Y.; Cao, H.; Sun, Z.: A MiniReview on Metal Recycling from Spent Lithium Ion Batteries. Engineering, 4 (2018), pp 361-370, https://doi.org/10.1016/j.eng.2018.05. 018

27. Gaines, L.: Lithium-ion battery recycling processes: Research towards a sustainable course, Susmat, 2018, e00068. https://doi.org/ 10.1016/j.susmat.2018.e00068

28. Ge, D.: Direct Lithium-Ion battery recycling to yield Battery grade cathode materials. Master-Thesis, 2019, https://vtechworks.lib.vt.edu/ handle/10919/92800 (20.04.2020)

29. Windisch-Kern, S.; Holzer, A.; Ponak, C.; Raupenstrauch, H.: Pyrometallurgical Lithium-lon-Battery Recycling: Approach to Limiting 
Lithium Slagging with the InduRed Reactor Concept, Processes, 9 (2021), p 84, https://doi.org/10.3390/pr9010084.

30. Gerold, E.; Luidold, S.; Honner, M.; Antrekowitsch, H.; Arnberger, A. Entwicklung eines hydrometallurgischen Recyclingkonzeptes für $\mathrm{Li}$ thium-Ionen-Batterien. Recy \& DepoTech 2020, 18.-20. November 2020, Montanuniversität Leoben. In Pomberger Roland; Adam Jo- sef; Aldrian Alexia; et al (Hrgb.): Konferenzband zur 15. Recy DepoTech-Konferenz, 2020

Hinweis des Verlags. Der Verlag bleibt in Hinblick auf geografische Zuordnungen und Gebietsbezeichnungen in veröffentlichten Karten und Institutsadressen neutral. 